Original Research Article

\title{
Comparison of methods of submission of suspected adverse drug reactions to the ADR monitoring centers in India
}

\author{
Sachin Kumar Kuchya ${ }^{1 *}$, Sarita Shrivastava ${ }^{2}$
}

${ }^{1}$ Department of Pharmacology, ${ }^{2}$ ADR Monitoring Centre Department of Pharmacology, NSCB MC, Jabalpur, Madhya Pradesh, India

Received: 08 June 2017

Revised: 28 June 2017

Accepted: 29 June 2017

*Correspondence to:

Dr. Sachin Kumar Kuchya, Email: sachinkuchya@

yahoo.com

Copyright: (C) the author(s), publisher and licensee Medip Academy. This is an openaccess article distributed under the terms of the Creative Commons Attribution NonCommercial License, which permits unrestricted noncommercial use, distribution, and reproduction in any medium, provided the original work is properly cited.

\begin{abstract}
Background: Traditionally, paper based suspected ADR forms were the only way of submitting suspected ADR (sADR) data. Recently the mobile android based ADR reporting app@ $(A p p @)$ has also been developed and a copyright was granted to the author. This study is done to assess the two, viz. paper based and App based, methods of submission of sADR data.

Methods: The sADR data submitted to the ADR Monitoring Centre (AMC), at Department of Pharmacology, NSCB MC Jabalpur. There is no such scale to assess the completeness of suspected ADR data received by individual AMCs. Therefore, appropriate algorithm and scale for Completeness scoring of filled sADR forms was designed, the basic tenets were adhered. A set of 10 sADR forms, submitted by either method, were subjected to Independent assessment by 3 assessors, who were not part of this study. The scores were then subjected to analysis, which revealed minimal variation across the assessment. Hence, the scale was adopted for the study.
\end{abstract}

Results: A total of 403 sADR's submitted to our AMC, were screened and subjected to scoring for completeness. Upon screening, 96.2\% (257/267) sADR submitted via paper based sADR form and $100 \%$ (136/136) of those submitted via App stood valid, and hence included in the study. All the suspected ADR (sADR) submitted via ADR Reporting app were, complete. The sADR data submitted via ADR reporting app, had an average completeness score of $34.7 \pm 2.4$ while those submitted via paper based form had an average of $29.2 \pm 2.4$. The difference is highly significant on Wilcoxon two sample test $(\mathrm{p}<0.001)$ and Kolmogorov-Smirnov test $(\mathrm{p}<0.001)$.

Conclusions: Compared to traditional paper based system, the ADR reporting app based sADR submission, is a better method.

Keywords: ADR reporting app, NCC-PvPI, Pharmacovigilance, SRS, sADR

\section{INTRODUCTION}

Pharmacovigilance (PV) is defined as the science and activities relating to the detection, assessment, understanding and prevention of adverse effects and all other problems related to medicines. ${ }^{1}$

Globally Pharmacovigilance systems mainly rely on spontaneous reporting - in which, suspected adverse drug reactions (sADRs) are reported to a national coordinating centre by health care professionals (HCPs), manufacturers or patients.
'Spontaneous reports' are so-called because they arise during a clinician's normal diagnostic appraisal of a patient, the clinician drawing the conclusion that a drug may be implicated in the causality of the clinical event. As with all diagnoses the certainty of attribution will vary with the skill and experience of the doctor, what confirmatory tests may show, the natural history of the clinical event, and the existence of other plausible explanations. ${ }^{2}$

Spontaneous reporting of suspected ADRs is particularly useful in identifying rare or delayed reactions, as such a system enables medicines to be monitored throughout their lifetime. ${ }^{3}$ 
Spontaneous reporting systems (SRS) are the easiest to establish and the cheapest to run. Also it's used as main source for the benefit -risk ratio evaluation of any drug. ${ }^{4}$

The method was developed in the 1960s - in response to the delay of around 5 years in recognizing an association between exposure to thalidomide during pregnancy and the congenital limb deformity called phocomelia. ${ }^{5}$ Systematic spontaneous reporting of possible drug caused adverse effects began with the 'Yellow card system' in the UK in 1964. It was a medium for doctors to report their concerns on marketed drugs, thereby enhancing the limited premarketing clinical data on safety. Now 54 countries around the world have similar systems, and many warnings of adverse drug reactions and some deletions from the market have been made on the basis of such reports. ${ }^{2}$

International standards of ADR reporting have been developing since late 1980s, through the Council for the International Organisation of Medical Sciences (CIOMS) and International Conference on Harmonization (ICH). ${ }^{6}$

While the FDA's MedWatch office was designed to facilitate voluntary reporting of ADRs, the Joint Commission on the Accreditation of Healthcare Organizations (JCAHO) found that as few as $5 \%$ of all ADRs each year are reported to appropriate authorities if solely relying on voluntary reporting by medical personnel. ${ }^{7}$ The voluntary submission of sADR data is abysmally low, even in developed countries like USA.

In India, Pharmacovigilance Programme of India (PvPI), was launched in the country in 2010, to ensure the safety with Medications. Indian Pharmacopoeia Commission functions as National Coordination Centre (NCC-PvPI) for the same. ${ }^{8}$ HCPs report sADR to any one of the ADR monitoring centres (AMC), situated all over the country. The sADR data submitted to the individual AMC's, is uploaded manually on the online National drug safety database platform, VIGIFLOW as Individual Drug Safety report (ICSR). Once submitted, the data goes into the Global drug safety database, VIGIBASE.

In year 2013, India's contribution to WHO-UMC's global drug safety database (Vigibase) was $2 \%$. India was 7 th in position among top 10 counties contributing to global drug safety database. Among Asian countries, India is the only country having more than 0.1 million ICSRs in Vigibase. ${ }^{9}$ This shows lack of empathy and interest towards voluntary submission of SADR data, in our country with a population of 1.25 Billion plus.

Traditionally, paper based sADR forms were the only way of submitting SADR data, but recently the mobile android based ADR Reporting app(C (App) has been developed and a copyright (http://copyright.gov.in/frmStatusGenUser.aspx, diary no. 773/2016-CO/SW) has been granted to the author. ${ }^{4}$

The App@ facilitates paperless and instant submission of
sADR to the AMC. The AMCs, upon receiving the SADR data, download the pdf version of filled sADR form, which is same as paper based form, the only difference that it is filled via App(C.

This study is done to assess the two, viz. paper based and App@ based, methods of submission of sADR data.

\section{METHODS}

A search for assessing the completeness of sADR data, submitted with AMC was done, via various search engines and also from, NCC-PvPI and WHO- Uppsala Monitoring Centre, both. The search however, returned Zero results.

There are tools like VIGIGRADE, to identify the well documented individual case safety reports (ICSR), but no such scale to assess the completeness of sADR data received by individual AMCs, was found. ${ }^{10}$

Therefore, a scale for assessment of filled sADR forms was designed, the basic tenets which were adhered are as follows:

1. A filled sADR form will be subjected to assessment of completeness, only if it has some legible data entry in all the four sections (Valid report) else it will be discarded as an Invalid Report - Screening.

2. The sADR form has 4 sections, viz Patient Identifiers, Suspected Adverse reaction, Suspected Medication and Reporter Information. All the sections should be filled as per SOP - IPC/PvPI/GEN 001/ Revision 3/ 15-5-15, laid down by NCC-PvPI, and are equally important.

3. Each section will have same score (10), while assessment of their completeness.

4. Each section usually has subsections for filling in mandatory as well as Essentially required items. The weightage for individual data filled in mandatory fields and Essentially required items, will be in the ratio of 2:1.

5. The weightage of Individual fields shall be calculated by dividing the score allotted to each section, divided by total weightage of all fields, in that section. And henceforth, distributed to each field as per their mandatory or essentially required item, status.

The scale and algorithm, is depicted in Figure 1.

Once the scale was finalized, a set of 10 sADR forms, submitted by either method, were subjected to Independent assessment by 3 assessors, who were not part of this study. The scores were then subjected to analysis, which revealed minimal variation across the assessment. Hence, the scale was adopted for the study.

The statistical tests which will be used are the nonparametric test like Wilcoxon two sample tests and 
Kolmogorov-Smirnov test for comparing the two frequency distributions for conclusion (Primary outcome)which method of submission brings in complete sADR data and is therefore better. Because the distribution of the completeness score of the sADR data submitted by either methods, can't be predicted therefore, it is safer to use nonparametric tests. ${ }^{11}$

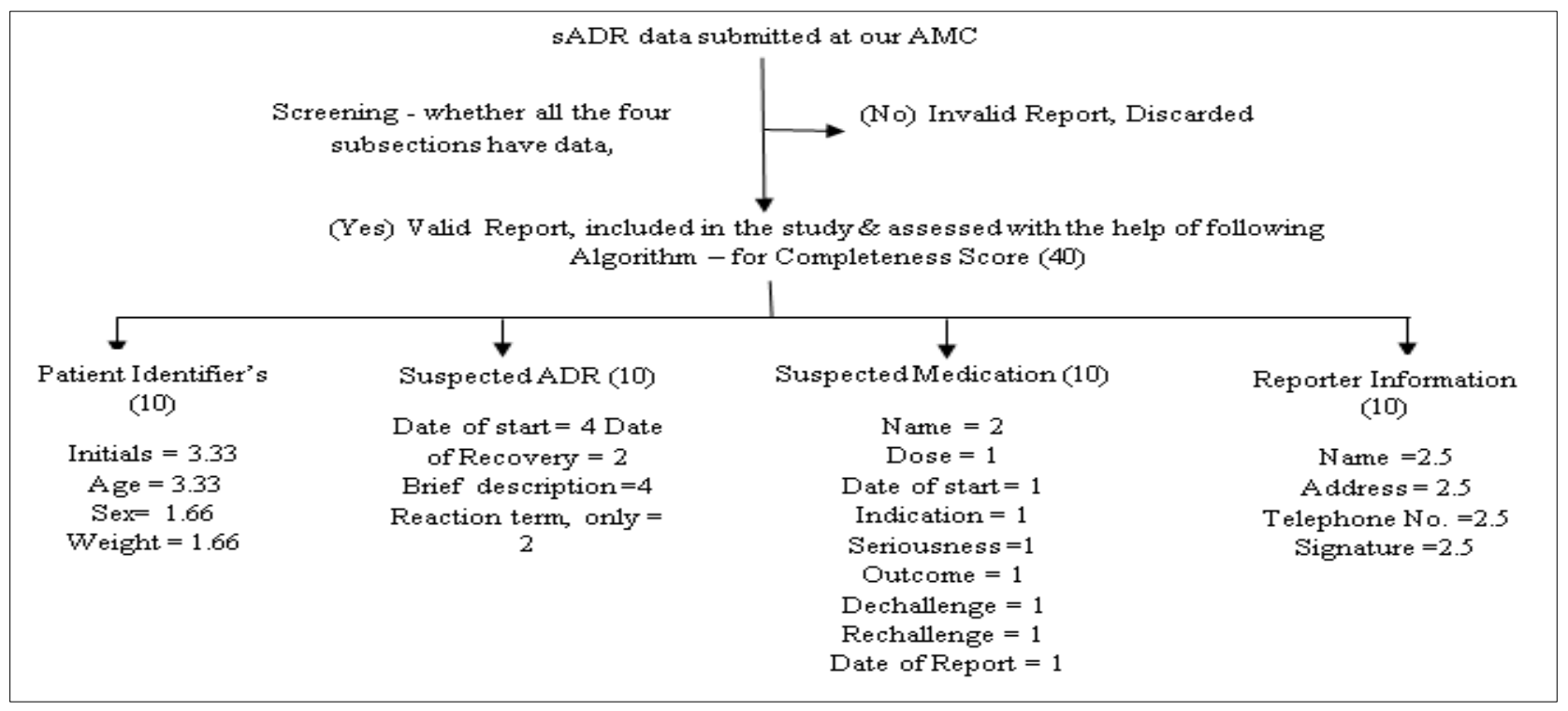

Figure 1: Original, the algorithm and scale finalized for screening and completeness scoring of sADR data submitted by respective methods.

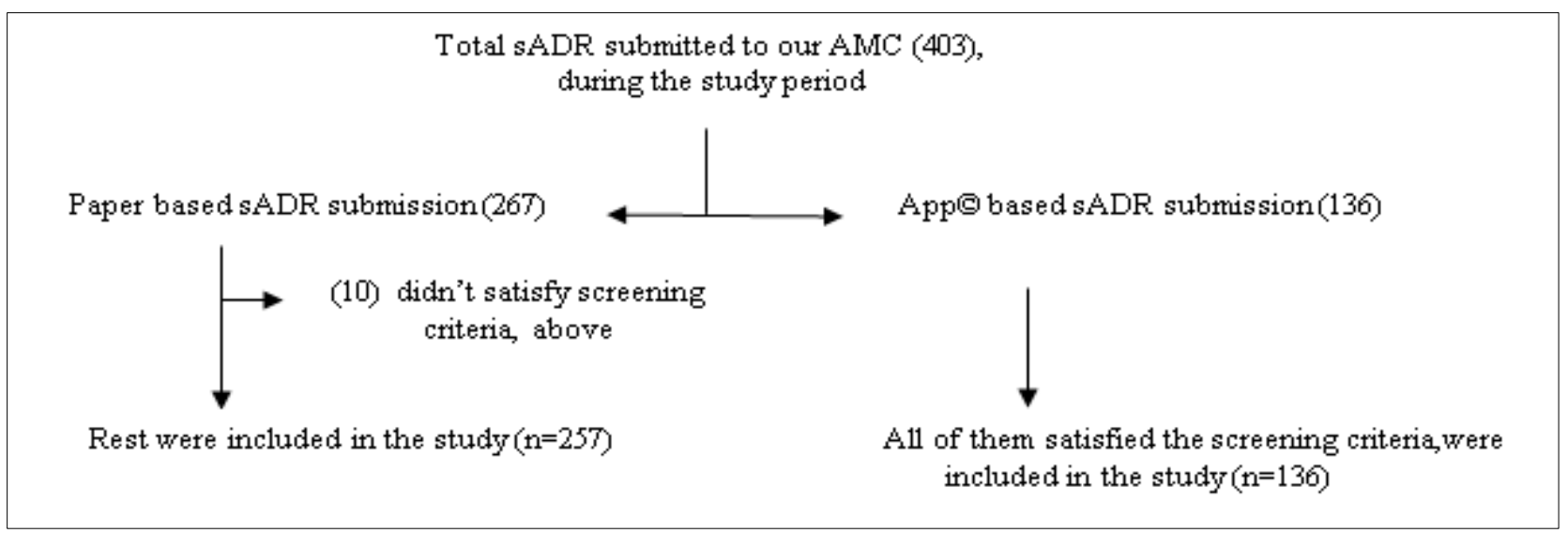

Figure 2: Original, depicting the outcome of screening of sADR data submitted by respective methods.

Data sets (the completeness score and section wise) obtained are unpaired. ${ }^{11}$ Therefore, Unpaired t tests (two tailed) will be used for ascertaining the significance in difference of means between the two methods of submission and between each of its sections (Secondary outcome). The sADR data submitted to our AMC, in between the period of $1^{\text {st }}$ Mar 15 till $31^{\text {st }}$ Jan 16 , were screened and subjected to scoring for completeness. A total of 403 sADRs were submitted during the abovesaid period, 267 were submitted via paper based sADR forms and 136 with the help of App@).
While screening, 10 sADR submitted with paper based form were excluded, as they didn't have Reporter Information and Suspected Adverse drug reaction. ${ }^{3,7}$ The rest, 257 stood valid, as per screening. Among those submitted via App@, all 136 were valid and included in the study. The consort diagram was as Figure 2.

\section{RESULTS}

In the first step while screening as per methodology enunciated above, around 96.2\% (257/267) sADR submitted via paper based SADR form could be included 
in the study while $100 \%$ (136/136) of those submitted via Appœ stood Valid, and hence, included in the study.

\section{Analysis}

The sADR data $(136 / 136,100 \%)$ submitted via App@ were valid and complete.

Table 1: Original, depicting the various measures of central tendency and spread of completeness scores, of sADR data submitted by respective methods.

\begin{tabular}{|lll|}
\hline $\begin{array}{l}\text { Method of } \\
\text { submission, thru }\end{array}$ & $\begin{array}{l}\text { ADR reporting } \\
\text { AppC }\end{array}$ & $\begin{array}{l}\text { Paper based } \\
\text { sADR form }\end{array}$ \\
\hline N & 136 & 257 \\
\hline Mean & 34.7 & 29.2 \\
\hline SD & 2.4 & 2.4 \\
\hline Median & 35.5 & 28.5 \\
\hline Minimum & 25.8 & 20.8 \\
\hline p25 & 33.0 & 27.5 \\
\hline p75 & 37.5 & 30.5 \\
\hline Maximum & 37.5 & 40.0 \\
\hline IQR & 4.5 & 3.0 \\
\hline
\end{tabular}

As is evident from the data (Table 1), the sADR data submitted via App $@$, had an average completeness score of $34.7 \pm 2.4$ while those submitted via paper based form had an average of 29.2 \pm 2.4 . Median score by App $\odot$ based is significantly higher than median score by paper based submission. This is further illustrated in the adjoining scatter diagram (Figure 3).

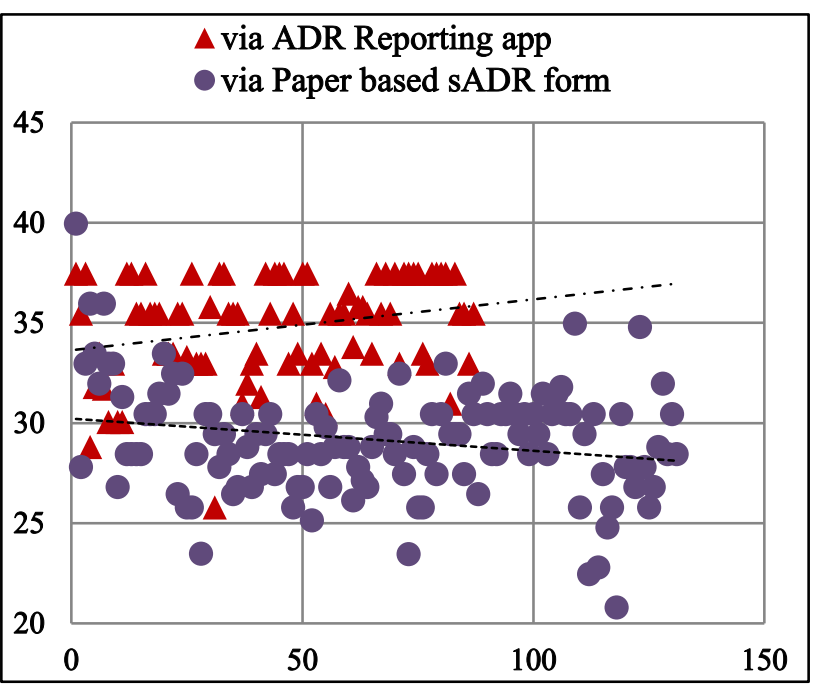

Figure 3: Original scatter figure, depicting completeness scores (on $\mathrm{Y}$ axis) of sADR data submitted by respective methods.

The difference is highly significant as per non parametric tests like, Wilcoxon two sample test $(\mathrm{p}<0.001)$ and Kolmogorov-Smirnov test for two frequency distributions $(\mathrm{p}<0.001)$. Therefore, the submission of sADR data via App $\odot$ is better.
Table 2: Original depicting the significance in difference of means between the two methods of submission, as a whole and section wise.

\begin{tabular}{|llll|}
\hline & $\begin{array}{l}\text { How big is the } \\
\text { difference, } \\
\text { Means } \pm \text { SD } \\
\text { (CI, 95\%) }\end{array}$ & $\begin{array}{l}\text { Unpaire } \\
\text { d t test, } \\
\text { two } \\
\text { tailed }\end{array}$ & $\begin{array}{l}\text { Are means } \\
\text { significantly } \\
\text { different? }\end{array}$ \\
\hline $\begin{array}{l}\text { Method of } \\
\text { submission }\end{array}$ & $\begin{array}{l}5.5 \pm 0.25 \\
(5.1-6.0)\end{array}$ & $\mathrm{p}<0.05$ & Yes \\
\hline $\begin{array}{l}\text { Patient } \\
\text { identifier }\end{array}$ & $\begin{array}{l}0.1 \pm 0.08 \\
(-0.05-0.25)\end{array}$ & $\begin{array}{l}\mathrm{P}= \\
0.1870\end{array}$ & No \\
\hline $\begin{array}{l}\text { Suspected } \\
\text { ADR }\end{array}$ & $\begin{array}{l}0.8 \pm 0.1 \\
(0.6-1.1)\end{array}$ & & \\
\cline { 1 - 2 } $\begin{array}{l}\text { Suspected } \\
\text { medication }\end{array}$ & $\begin{array}{l}1.1 \pm 0.11 \\
(0.93-1.3)\end{array}$ & $\mathrm{p}<0.0001$ & Yes \\
\cline { 1 - 2 } $\begin{array}{l}\text { Reporter } \\
\text { Information }\end{array}$ & $\begin{array}{l}3.6 \pm 0.15 \\
(3.3-3.9)\end{array}$ & \\
\hline
\end{tabular}

\section{DISCUSSION}

The submission of sADR data via App $\subset$ is better and should be a preferred mode of submission of sADR.

A higher average and median values are seen with completeness score of sADR data submitted by App ( based method. This can be inferred as that the App@ based submission brings in Complete and more of data, which will add more meaning to the follow up activity.

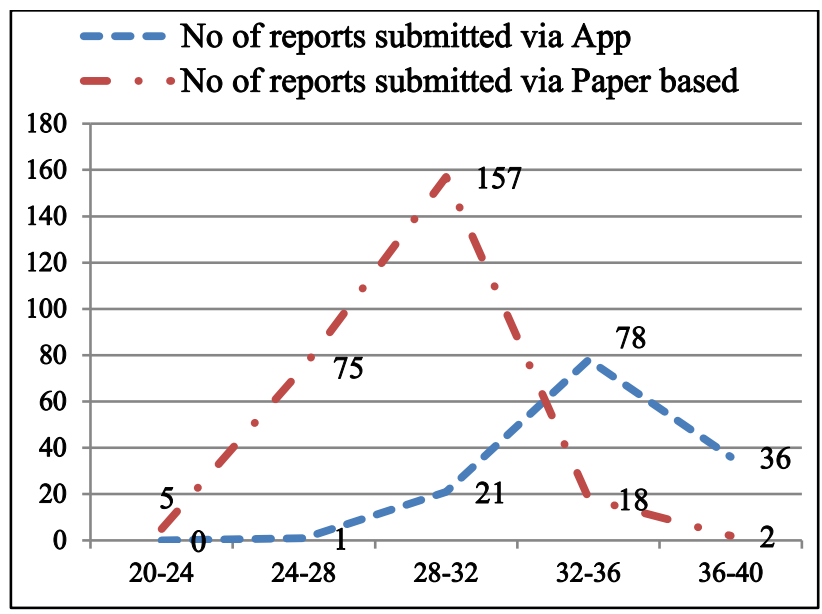

No. of sADR reports (on $\mathrm{Y}$ axis) submitted in each range of completeness score (on $\mathrm{X}$ axis).

Figure 4: Original, the frequency distribution of completeness scores of SADR data submitted by respective methods.

Figure 4 clearly shows that the 2 distributions are different. Paper based sADR data submission has an approximately normal distribution, but App(C) based submission is left skewed. This can be inferred as, a greater likelihood of submission of complete sADR data (hence, with higher completeness score) when App@ is used.

The difference is significant for methods of submission $(\mathrm{p}<0.05)$ and highly significant for all the sections 
$(\mathrm{p}<0.0001)$ except for patient Identifier's $(\mathrm{p}=0.1870)$ (Table 2). This could be because the patient identifier section, has less no. of mandatory fields, has a check box need's much less data, is the first section therefore filled satisfactorily by either method of submission.

Owing to certain validations, App@ $@$ based reports can only be submitted, once all the mandatory data has been entered, therefore only valid reports can be submitted (Figure 2).

The Reporter Information need not be filled repeatedly, therefore will always be complete and verifiable (Table 2), therefore each and every SADR data submitted can be followed up, too. Repeat submissions by same HCP, will have a higher completeness score and the ease of submission will progressively increase voluntary sADR reporting.

As already on record, the problem of communication gap between input operator and output analyzer is a major issue. $^{12}$ The App(C) applies bilateral standardization and helps in overcoming this communication gap.

It enhances the Knowledgebase of and enriches the reporter, via an Objective and Rationale, built-in algorithm for Causality assessment based upon WHO criteria, which depicts the strength of association between the reporter's observation and suspected drug.

The practice of mandatory reporting in Sweden and Recent discussions in Canada have raised the prospect of federal legislation for mandatory reporting of ADRs. ${ }^{7}$ If such an amendment is introduced in our country, heretofore relying solely on voluntary reporting, this App@ would also avoid infrastructural gap.

Other salient observations were:

- Criteria for seriousness and outcomes were clearly marked in sADR submitted via App@, while they were often not clear, overwritten, multiple options are marked, or left unmarked in SADR data submitted via paper based forms.

- $\quad$ The drug names, time lines were very clear in sADR submitted via App $\odot$, while in paper based reports, very often some poorly scribbled abbreviations, poor and often distorted timelines esp concerning, date of report were the norm.

- The App@ $\odot$ is user friendly and involves the HCP's feedback in causality assessment. This will help by enhancing their participation and uniformity in causality assessment.

The certain disadvantages are signatures cannot be placed, follow up of previously reported cases need to filled afresh, currently the App@ $\odot$ is available only on Android based mobile platform, the other versions for iOS, Lumia and desktop versions too need to be built upon.
The voluntary submission of sADR data, is abysmally low. ${ }^{7,10}$ Nationwide promotion and utilization of the App(C) will improve the Quality as well as the volume of sADR data submission, by almost 100-1000 times, which shall reflect as an Increase in Indian Contribution in global drug safety database, currently it stands close to $1.6-2.0 \%$ (Uppasla Reports 66-72 https://www.whoumc.org/media/1691/28198.pdf), while we account for over $16 \%$ of world population.

\section{CONCLUSION}

The App(C, is a better method for submission of sADR data. It also has ability to bring in legible complete, good quality data. Has more rationale and scientific, causality assessment and a verifiable reporter, therefore can be followed up easily. It should be promoted, as it makes sADR data submission paperless and Instant, too.

Funding: No funding sources

Conflict of interest: None declared

Ethical approval: Not required

\section{REFERENCES}

1. WHO. The importance of pharmacovigilance: safety monitoring of medicinal products. Geneva: WHO; 2002.

2. Ralph Edwards I. Spontaneous reporting of what? Clinical concerns about drugs. British Journal of Clinical Pharmacology. 1999;48(2):138-41.

3. Bandekar MS, Anwikar SR, Kshirsagar NA. Quality check of spontaneous adverse drug reaction reporting forms of different countries. pharmacoepidemiology and drug safety; 2010. Published online in Wiley Online Library. Available from: wileyonlinelibrary.com.

4. Kuchya S, Kalaiselvan V, Kaur I, Singh GN. Mobile application an approach to enhance easy adverse drug reactions reporting in India. Health and Technology. 2016 Jul 1;6(2):157-8.

5. Waller PC. Making the most of spontaneous adverse drug reaction reporting. Basic \& Clinical Pharmacology \& Toxicology. 2006 Mar 1;98(3):3203.

6. Bahri P, Tsintis P. Pharmacovigilance-related topics at the level of the International Conference on Harmonisation (ICH). Pharmacoepidemiology and drug safety. 2005 Jun 1;14(6):377-87.

7. Carleton BC, Smith MA. Drug safety: Side effects and mistakes or adverse reactions and deadly errors?. British Columbia Medical Journal. 2006 Sep;48(7):329-33.

8. Vivekanandan K, Rishi K, Prasad T, Arunabh T, Singh GN. Status of documentation grading and completeness score for Indian individual case safety reports. Indian J Pharmacol. 2015;47:325-7.

9. Lihite RJ, Lahkar M. An update on the Pharmacovigilance Programme of India. Frontiers in Pharmacology. 2015;6:194. 
10. Bergvall T, Lindquist M, G. Nore N. VigiGrade: A Tool to Identify Well-Documented Individual Case Reports and Highlight Systematic Data Quality Issues Drug Saf. 2014;37:65-77.

11. Nayak BK, Hazra A. How to choose the right statistical test? Indian Journal of Ophthalmology. 2011;59(2):85-6.
12. Fletcher AP. Spontaneous adverse drug reaction reporting vs event monitoring: a comparison. Journal of the Royal Society of Medicine. 1991;84(6):341-4.

Cite this article as: Kuchya SK, Shrivastava S. Comparison of methods of submission of suspected adverse drug reactions to the ADR monitoring centers in India. Int J Basic Clin Pharmacol 2017;6:2032-7. 